Research Article

\title{
Comparison of the Initiation Time of Enteral Nutrition for Critically Ill Patients: At Admission vs. 24 to 48 Hours after Admission
}

\author{
Anshan Yu, Yanmei Xie, Meixin Zhong, Fen Wang, Huachun Huang, Liang Nie, \\ Xiaofeng Liu, Mingfang Xiao, and Hongquan Zhu $\mathbb{C}$ \\ Department of Critical Care Medicine, The First Affiliated Hospital of Gannan Medical University, Ganzhou, \\ Jiangxi 341000, China \\ Correspondence should be addressed to Hongquan Zhu; zhuhongquan@21cn.com
}

Received 2 July 2021; Accepted 6 September 2021; Published 18 September 2021

Academic Editor: Piergiorgio Fedeli

Copyright (c) 2021 Anshan Yu et al. This is an open access article distributed under the Creative Commons Attribution License, which permits unrestricted use, distribution, and reproduction in any medium, provided the original work is properly cited.

\begin{abstract}
Objective. To investigate the better time of initiation of enteral nutrition for critically ill patients, such as at admission or 24 to 48 hours after admission. Methods. This was a prospective, randomized, parallel-controlled, single-blind, interventional clinical trial. A total of 100 patients admitted to the intensive care unit (ICU) of our hospital between January 2017 and December 2018 were recruited in this study. These patients had been divided into the control group or intervention group by a computer-generated random number table, and each group had 50 patients. For the control group, a gastric tube was inserted to start enteral nutrition at 24 to 48 hours after admission. For the intervention group, a nasojejunal tube was placed to start enteral nutrition at admission. The main endpoints included serum albumin and prealbumin at admission and on days 3, 7, and 14 after admission, length of ICU stay, ventilator time, and complications such as diarrhea, gastric retention, esophageal reflux, and pulmonary infection. Results. The results showed that serum albumin and prealbumin were significantly higher in the intervention group than in the control group $(P<0.05)$. The length of ICU stay $(P<0.05)$ and ventilator time $(P<0.05)$ were both significantly shorter in the intervention group than in the control group. The incidences of gastric retention, esophageal reflux, and pulmonary infection were significantly lower in the intervention group than those in the control group $(P<0.05)$. Conclusion. In the absence of contraindications, enteral nutrition can be initiated immediately after admission to the ICU (within 6 hours), and feeding nasojejunal tube is recommended. It can improve the nutritional status and prognosis of critical patients, improve the feeding effect, shorten the length of stay in the ICU and the use of the ventilator, and reduce the incidence of complications.
\end{abstract}

\section{Introduction}

Insufficient nutrition is very common in critically ill patients admitted to the intensive care unit (ICU), and it is important to strengthen nutritional support for these patients. Enteral nutrition is the first choice of nutritional support in critically ill patients $[1,2]$. For these patients, early enteral nutrition is critical for restoring normal intestinal permeability, preventing intestinal infection and intestinal failure, and improving outcomes $[3,4]$. The initiation time and feeding route of enteral nutrition are the focus of current research. Regarding the time of initiation of enteral nutrition, most current guidelines and studies recommend that enteral nutrition be initiated at 24 hours after admission [5-7]. However, studies have shown that this regimen still leads to feeding delays.

In a multicenter study, the average enteral nutrition initiation time in ICU patients was 46.5 hours [8]. Delayed feeding leads to insufficient intake and malnutrition in patients, which further leads to poor prognosis and increased mortality $[9,10]$. Several previous studies have investigated the benefits of earlier enteral nutrition [11-13]; in these studies, enteral nutrition was initiated as early as 4 hours after admission. However, the studies were conducted in patients 
with obstructive jaundice and burns, respectively, and did not include other critically ill patients. Therefore, the study calls for improving patient feeding to be the focus of quality care in the ICU [14].

This study was designed to investigate the initiation of enteral nutrition at admission versus at 24 to 48 hours after admission in critically ill adult patients, so as to find the best enteral nutrition initiation time and nutrition pathway for patients with severe illness, reduce feeding delay, and improve nutrition and prognosis of patients with severe illness.

\section{Materials and Methods}

2.1. Study Design. This was a prospective, randomized, parallel-controlled, single-blind, interventional clinical trial.

2.2. Subjects. From January 2017 to December 2018, patients admitted to the intensive care unit (ICU) of our hospital were enrolled in this study. These patients had been divided into the control group or intervention group by a computergenerated random number table. For the control group, a gastric tube was inserted to start enteral nutrition at 24 to 48 hours after admission. For the intervention group, a nasojejunal tube was placed to start enteral nutrition at admission. This study was conducted in accordance with the Declaration of Helsinki and approved by the ethics committee of our hospital. All participants had signed informed consent.

\subsection{Inclusion and Exclusion Criteria and Group Assignment.} The inclusion criteria were as follows: (1) patients admitted to the general adult ICU; (2) patients who were $\geq 14$ years of age; (3) patients who have signed informed consent.

The exclusion criteria were as follows [5]: (1) hemodynamic instability (mean arterial pressure <60 mm Hg); (2) contraindications for enteral nutrition due to functional, structural, or physiological gastrointestinal disorders such as intestinal ischemia or obstruction, gastrointestinal perforation or fistula, abdominal compartment syndrome, or active upper gastrointestinal bleeding; (3) other contraindications for enteral nutrition, such as selective enteroscopy or bowel surgery.

2.4. Calculation of Sample Size. Sample size was calculated as $G^{*}$ power (two independent sample $t$-tests, two-sided, significance level of 0.05 , statistical power of 0.8 , and effect size of 0.6217 calculated with means and deviations from the two groups in the pilot study). The result showed that 84 subjects were required, and 100 subjects should be enrolled considering a $20 \%$ exclusion rate.

2.5. Group Assignment. A computer-generated random number table was used for randomization. The patients were assigned an ID number from 1 to 100 based on the time of admission, and SPSS v 25.0 was used to generate a random number associated with each patient ID. Patients with a random number from 1 to 50 were assigned to the intervention group, and those with a number from 51 to 100 were assigned to the control group, resulting in 50 patients in each group. The randomization process was performed by a designated member of the study team. The assignment results were sealed in an opaque envelope and were not disclosed to the data collection staff.

\subsection{Interventions}

2.6.1. Tube Materials. A silicone enteral nutrition tube with a guide wire (F12) was used in both groups. The tube length was $80 \mathrm{~cm}$ in the control group and $145 \mathrm{~cm}$ in the intervention group, with $20 \mathrm{~cm}$ of extra length.

2.6.2. Training. A tube-insertion nurse team and an observation nurse team were established and trained. The two teams were independent of each other and had different members. The first team inserted the tube; and the second team, which was blinded to group assignment, recorded all outcome measures.

2.6.3. Tube Insertion and Management. In the control group, the tube was inserted into the stomach, with a length of about $60 \mathrm{~cm}$ from the nose to the stomach 24 to 48 hours after admission (insertion length: $60 \mathrm{~cm}$, extra: $20 \mathrm{~cm}$; both segments were labeled "enteral nutrition tube"). For the intervention group, a tube was inserted into the duodenum with a length of $125 \mathrm{~cm}$ from the nose to the duodenum (no more than 6 hours after admission) (insertion length: $125 \mathrm{~cm}$ (confirmed with ultrasound to have passed the pylorus) [15], extra: $20 \mathrm{~cm}$; both segments were labeled "enteral nutrition tube"). Both groups received routine enteral nutrition management. After insertion, the observation nurses provided management and recorded all outcome measures. For patients with no contraindications, the head of the bed was raised by $30^{\circ}$. Daily enteral nutrition (Nutrison Fibre, Nutricia, Netherlands, $1 \mathrm{kcal} / \mathrm{mL}$ ) was started at $20 \mathrm{~mL} / \mathrm{h}$ $(20 \mathrm{kcal} / \mathrm{h})$ and infused at a constant rate with a nutrition pump. The gastric contents were aspired every 4 hours. If the volume of the aspired contents was less than $150 \mathrm{~mL}$ or less than the volume of infusion over 4 hours, the infusion rate was increased by $20 \mathrm{~mL} / \mathrm{h}$ until the feeding target $(30 \mathrm{kcal} /$ $\mathrm{kg} / \mathrm{d}$ ) was reached in 2 days. If the aspired contents were no less than $150 \mathrm{~mL}$ or the volume of infusion over 4 hours (indicating gastric retention) or if diarrhea and vomiting occurred, the infusion rate was reduced by $50 \%$. After 4 hours, if the volume of the aspired gastric content was still no less than the volume infused over 4 hours or if vomiting persisted, feeding was terminated.

2.7. Outcome Measures. The primary outcome measures included serum albumin and prealbumin at admission and on days 3, 7, and 14 after admission. The secondary outcome measures included the length of ICU stay, ventilator time, and complications during the first 14 days in the ICU.

The complications included diarrhea, reflux aspiration, gastric retention, and pulmonary infection. The complications were evaluated as follows [16]: (1) diarrhea: if the frequency of bowel movements was equal to or greater than 
4 per day or was increased by 2 times per day, diarrhea was considered; (2) gastric retention: gastric retention was considered if the volume of the aspired gastric contents (every 4 hours) was no less than $150 \mathrm{~mL}$ or no less than the volume infused over 4 hours; (3) pulmonary infection: pulmonary infection was considered if there were new signs of inflammation on the chest X-ray with any two of the following symptoms: cough, breathing difficulty, fever, leukocytosis, wheezing, and rales per auscultation.

2.8. Statistical Analysis. We used the software program SPSS 25.0 (IBM, Chicago, USA) to conduct the statistical analysis. The continuous variables of the normal distribution were expressed as mean \pm standard deviation, the continuous variables of the nonnormal distribution were expressed as median (interquartile range (IQR)), and the categorical variables were expressed as frequency (percentage (\%)). The Kolmogorov-Smirnov (KS) test was used to analyze the normality of continuous data. Normally distributed data were analyzed with the two independent sample $t$-tests; nonnormally distributed data were analyzed with Wilcoxon's rank-sum test. Categorical data were analyzed with the $\chi^{2}$ test. For repeated measurements, normally distributed data were analyzed with the analysis of variance of repeated measurements and the general linear model (processing effect, time effect, and the interaction between the two); nonnormally distributed data were analyzed with generalized estimating equations. $P<0.05$ was considered statistically significant.

\section{Results}

3.1. Drop-Out and Exclusion Criteria. A total of 100 patients had been recruited. However, a total of 5 patients were withdrawn from the study due to less than 72 hours of hospital stay, including 3 in the control group and 2 in the intervention group. A total of 8 patients were withdrawn from the study due to intravenous albumin infusion during the observation period, including 4 in the control group and 4 in the intervention group. Finally, 44 patients in the intervention group and 43 in the control group $(N=87)$ completed the study.

3.2. The General Characteristics. A total of 87 patients who were admitted to the ICU of our hospital between January 2017 and December 2018 were enrolled in this study, including 44 patients in the intervention group and 43 patients in the control group. There were 59 males and 28 females, aged 15 to 88 years (mean: $56.8 \pm 16.8$ ). No significant between-group differences were observed in disease composition and age, sex (Table 1), or baseline data such as APACHE II score at admission, albumin at admission, and prealbumin level at admission (Table 2).

In consideration of the influence of etiology on the outcome, we analyzed the etiology of the two groups of patients, and the results showed (see Table 1) that there was no difference in etiology between the control group and the intervention group. In order to analyze the effects of different disease severities and different intestinal functions on the outcome indicators, we compared the two groups, and the results showed (see Table 2) that there were no differences between the control group and the intervention group in disease severity, preintervention albumin, and prealbumin.

\subsection{Changes in Mean Albumin over Time between Two} Groups. The results showed that the albumin level was initially similar between the two groups. Over time, the value increased in the intervention group, while it decreased and then remained low in the control group. Mauchly's sphericity test showed that, for the interaction item treatment * time, the dependent variable did not comply with the sphericity hypothesis $\left(\chi^{2}=22.379, P \leq 0.001\right.$, and Greenhouse-Geisser epsilon $=0.508$ ) and thus required Greenhouse-Geisser correction. The details are shown in Figure 1.

The results showed that the corrected interaction effect of treatment * time was $F(1.524,22.855)=3.033(P=0.08)$, indicating no statistical significance or the absence of an interaction, and the main effect of treatment on albumin was statistically significant $(P=0.039)$ (Table 3$)$. The albumin level was higher in the intervention group than in the control group by $2.761 \mathrm{~g} / \mathrm{L}$ (95\% confidence interval (CI): $0.157-5.365)$. There was no significant effect on albumin $(P=0.809)$.

\subsection{Changes in Prealbumin Levels between Two Groups.} Based on the intercept $(0.187, P<0.001)$ parameter estimate and test, the mean initial prealbumin level was significantly higher than zero in the two groups. In the intervention group, the prealbumin level changed at a rate of 0.002 over time, indicating a slight but insignificant increase in the prealbumin level $(P=0.148)$. The prealbumin level was higher in the intervention group than in the control group by $0.048 \mathrm{mg} / \mathrm{L}(P=0.002)$. No significant interaction effect was observed between the treatment time and group $(P=0.598)$. The details are listed in Table 4.

3.5. Length of ICU Stay and Ventilator Time between Two Groups. In the intervention group, the length of ICU stay was $6.4(3.3,10.7)$ days, and the ventilator time was $3.6(1.2,6.0)$ days. In the control group, the length of ICU stay was 7.9 (5.6, $12.1)$ days, and the ventilator time was $5.5(4.1,8.8)$ days. The results showed that the length of ICU stay and ventilator time were significantly shorter in the intervention group than in the control group $(P<0.05)$. The details are listed in Table 5 .

3.6. Complications between Two Groups. In the control group, 5 cases $(11.6 \%)$ had diarrhea, 5 cases $(11.6 \%)$ had gastric retention, and 7 cases $(16.3 \%)$ had pulmonary infection. In the intervention group, 2 cases $(4.5 \%)$ had diarrhea, no case $(0.0 \%)$ had gastric retention, and 1 case (2.3\%) had pulmonary infection. The results showed that no significant between-group difference was observed in the incidence of diarrhea. The incidences of gastric retention and pulmonary infection were significantly lower in the intervention group than in the control group $(P<0.05)$. The details are listed in Table 6. 
TABle 1: Disease composition $(n, \%)$.

\begin{tabular}{|c|c|c|c|c|c|c|}
\hline Diagnosis & Cerebral infarction with respiratory failure & AECOPD & Postbrain surgery & Multitrauma & Others & Total \\
\hline Observation group & $15(34.1)$ & $7(15.9)$ & $8(18.2)$ & $7(15.9)$ & $7(15.9)$ & $44(100)$ \\
\hline Control group & $8(18.6)$ & $14(32.6)$ & $9(20.9)$ & $3(7.0)$ & $9(20.9)$ & $43(100)$ \\
\hline$x^{2}$ & \multicolumn{6}{|c|}{6.362} \\
\hline$P$ & \multicolumn{6}{|c|}{0.174} \\
\hline
\end{tabular}

TABLE 2: APACHE II score at admission, albumin level at admission, and prealbumin level at admission.

\begin{tabular}{|c|c|c|c|c|}
\hline Group & $n$ & APACHE II score at admission $( \pm s)$ & $\begin{array}{l}\text { Albumin level at admission } \\
( \pm \mathrm{s}, \mathrm{g} / \mathrm{L})\end{array}$ & Prealbumin level at admission $( \pm \mathrm{s}, \mathrm{g} / \mathrm{L})$ \\
\hline Intervention group & 44 & $22.7 \pm 9.5$ & $35.33 \pm 6.58$ & $0.175 \pm 0.075$ \\
\hline Control group & 43 & $22.2 \pm 6.1$ & $36.19 \pm 6.80$ & $0.153 \pm 0.069$ \\
\hline$\chi^{2} / t$ & & 0.276 & 0.605 & 1.455 \\
\hline$\stackrel{R}{P}$ & & 0.783 & 0.547 & 0.149 \\
\hline
\end{tabular}

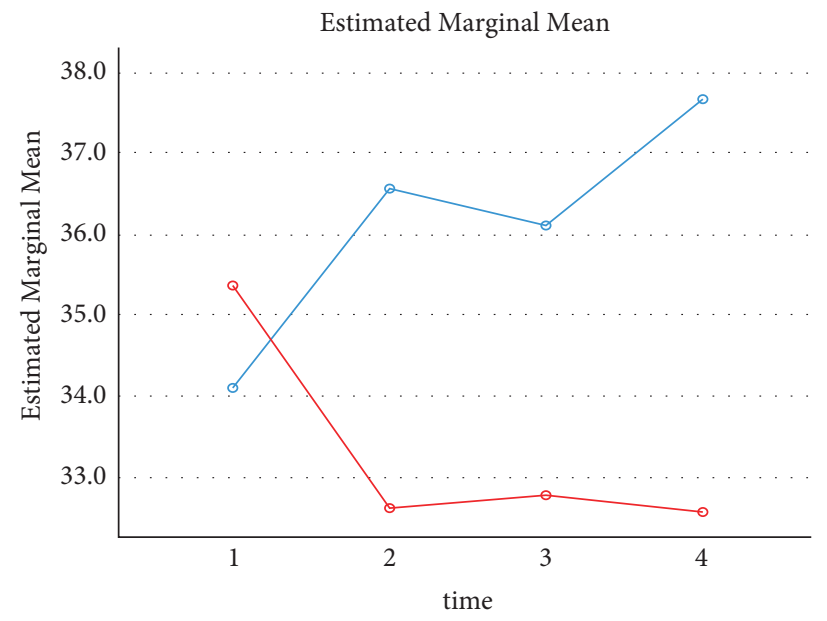

treatment

Observation group
Control group

FIGURE 1: Line chart of the changes in mean albumin over time.

TABle 3: Tests of within-subject effects.

\begin{tabular}{llcccc}
\hline & Source & Type III sum of squares & $\mathrm{d} f$ & Mean squares & $F$ \\
\hline Treatment & Greenhouse-Geisser & 243.929 & 1.000 & 243.929 & 5.107 \\
Error (treatment) & Greenhouse-Geisser & 716.460 & 15.000 & 47.764 & 0.039 \\
time & Greenhouse-Geisser & 7.965 & 1.429 & 5.572 & 0.130 \\
Error (time) & Greenhouse-Geisser & 918.557 & 21.441 & 42.841 & 0.809 \\
treatment ${ }^{*}$ time & Greenhouse-Geisser & 184.693 & 1.524 & 121.216 & 3.033 \\
Error (treatment* time) & Greenhouse-Geisser & 913.303 & 22.855 & 39.961 & \\
\hline
\end{tabular}

TABLE 4: GEE model parameter estimates of prealbumin.

\begin{tabular}{lcccccc}
\hline Parameter & $B$ & Standard error & $95 \%$ CI & Wald $\chi^{2}$ & $\mathrm{~d} f$ & $P$ \\
\hline Intercept & 0.187 & 0.012 & 0.164 to 0.210 & 254.789 & 1 & 0.000 \\
Group & 0.048 & 0.016 & 0.078 to 0.017 & 9.372 & 1 \\
Measure time & 0.002 & 0.001 & -0.001 to 0.004 & 2.093 & 0.002 \\
Group* measure time & 0.001 & 0.002 & -0.002 to 0.004 & 0.278 & 1 \\
\hline
\end{tabular}


TABLE 5: Length of ICU stay and ventilator time ( $n, M$ (P25, P75), unit: day).

\begin{tabular}{lcr}
\hline Group & Length of ICU stay & Ventilator time \\
\hline Intervention & $44,6.4(3.3,10.7)$ & $31,3.6(1.2,6.0)$ \\
Control group & $43,7.9(5.6,12.1)$ & $30,5.5(4.1,8.8)$ \\
$Z$ & -2.238 & -2.179 \\
$P$ & 0.025 & 0.029 \\
\hline
\end{tabular}

TABLe 6: Complications $(n(\%))$.

\begin{tabular}{lccc}
\hline Group & Diarrhea & Gastric retention & Pulmonary infection \\
\hline Control group $(n=43)$ & $5(11.6)$ & $5(11.6)$ & $7(16.3)$ \\
Intervention group $(n=44)$ & $2(4.5)$ & $0(0.0)$ & $1(2.3)$ \\
$x^{2}$ & 0.266 & 0.026 & 0.030 \\
$P$ & & & \\
\hline
\end{tabular}

\section{Discussion}

Enteral nutrition refers to the provision of nutrients through the gastrointestinal tract to maintain the body's metabolism. The gastrointestinal tract plays an important role in immune defense. Compared with parenteral nutrition, enteral nutrition not only has the advantages of more physiologic absorption and utilization of nutrients but also helps maintain the integrity of the intestinal mucosal structure and barrier function.

Initiation delay is one of the causes of insufficient enteral nutritional intake in critically ill patients. The "Consensus of Early Enteral Nutrition Clinical Practice in Critically Ill Patients" [5] of 2018 states that enteral nutrition support should be provided as soon as possible after related contraindications are excluded. Clinical studies have shown that initiation delay is one reason why patients fail to reach the feeding target and that it has adverse effects on recovery $[14,17,18]$. One of the critical factors for ensuring that patients benefit from nutritional therapy is to reduce initiation delay in order to achieve the feeding target as soon as possible. Because of clinical practice and concerns about the poor tolerability of enteral nutrition by critically ill patients, clinicians often wait 24 hours after admission to decide whether to initiate enteral nutrition, which is the cause of initiation delay. Early initiation of enteral nutrition at admission reduces the observational wait time after admission, thereby reducing initiation delay and improving outcomes. This study showed that the initiation of enteral nutrition at admission improved serum albumin and prealbumin and reduced the length of ICU stay and ventilator time relative to the initiation of enteral nutrition at 24 to 48 hours after admission.

Due to technical and equipment limitations, via a nasogastric tube is still the most common method for providing early enteral nutritional support; however, sedative, analgesic, and vasoactive drugs are often used in critically ill patients, resulting in varying degrees of decreased gastric motility. Consequently, nasogastric feeding is associated with a high risk of aspiration and pulmonary infection. Its safety profile is less than satisfactory $[19,20]$. Several studies $[16,21-23]$ have shown that nasogastric feeding is associated with a high incidence of feeding intolerance, which leads to high morbidity and mortality and prolonged ICU stays, with adverse effects on patient recovery. Nasojejunal feeding reduces aspiration and pneumonia, improves outcomes and gastrointestinal tolerability, and reduces complications and the time to reach the feeding target $[24,25]$, which allows enteral nutrition to be initiated early and safely. Early nutritional intervention by nasojejunal feeding can reduce the incidence of complications.

Fuentes Padilla et al. [26] reported that it was uncertain whether early enteral nutrition, compared with delayed enteral nutrition, affects the risk of mortality within 30 days, feed intolerance or gastrointestinal complications, or pneumonia due to very low-quality evidence. Tian et al. [27] reported that early enteral nutrition reduced mortality and pneumonia compared with delayed enteral intake, but there were no clear clinical advantages of early enteral nutrition over parenteral nutrition. However, our study found that early enteral nutrition can improve the nutritional status and prognosis of critical patients, improve the feeding effect, shorten the length of stay in the ICU and the use of the ventilator, and reduce the incidence of complications. In summary, for critically ill patients who require early enteral nutrition support and have no contraindications, enteral nutrition should be initiated immediately at admission (within 6 hours). Early nutritional intervention by nasojejunal feeding is advantageous for improving nutrition status, reducing the lengths of ICU stays and ventilator time, and promoting recovery.

This study still had several limitations: firstly, this was a small $(N=87)$, single-center study. Large multicenter studies are needed to further validate the results. Secondly, in this study, the measures were observed only through day 14 after admission, and there was no observation of long-term effects. Studies with a longer observation period are needed to further validate the results.

\section{Conclusion}

In the absence of contraindications, enteral nutrition can be initiated immediately after admission to the ICU (within 6 hours), and feeding nasojejunal tube is recommended. It can improve the nutritional status and prognosis of critical patients, improve the feeding effect, shorten the length of 
stay in the ICU and the use of the ventilator, and reduce the incidence of complications.

\section{Data Availability}

The datasets used or analyzed during the current study are available from the corresponding author upon reasonable request.

\section{Ethical Approval}

This study was conducted in accordance with the Declaration of Helsinki and approved by the ethics committee of our hospital.

\section{Consent}

All participants signed informed consent.

\section{Conflicts of Interest}

All authors declare no conflicts of interest.

\section{Authors' Contributions}

Yu AS, Xie YM, and Zhu HQ conceptualized and designed the research, wrote the manuscript, and critically revised the manuscript for intellectual content. Zhong MX and Wang F contributed to the acquisition of the data. Huang $\mathrm{HC}$ and Nie L analyzed and interpreted the data. Liu XF and Xiao MF performed statistical analysis. All authors approved the final version to be published.

\section{Acknowledgments}

This research was supported by the Science and Technology Support Plan Fund of Science and Technology Department of Jiangxi Province, China (Grant no. 20151BBG70081).

\section{References}

[1] R. Bankhead, J. Boullata, S. Brantley et al., "A.S.P.E.N. enteral nutrition practice recommendations," Journal of Parenteral and Enteral Nutrition, vol. 33, no. 2, pp. 122-167, 2009.

[2] K. G. Kreymann, M. M. Berger, N. E. P. Deutz et al., "ESPEN guidelines on enteral nutrition: intensive care," Clinical Nutrition, vol. 25, no. 2, pp. 210-223, 2006.

[3] D. K. Heyland, "Nutritional support in the critically ill patient," Critical Care Clinics, vol. 14, no. 3, pp. 423-440, 1998.

[4] M. J. Cangelosi, H. R. Auerbach, and J. T. Cohen, "A clinical and economic evaluation of enteral nutrition," Current Medical Research and Opinion, vol. 27, no. 2, pp. 413-22, 2011.

[5] R. H. Sun, R. L. Jiang, and M. Huang, "Consensus of early enteral nutrition clinical practice in critically ill patients," Chinese Critical Care Medicine, vol. 30, no. 8, pp. 715-721, 2018.

[6] S. A. McClave, B. E. Taylor, R. G. Martindale et al., "A Guidelines for the provision and assessment of nutrition support therapy in the adult critically ill patient," Journal of Parenteral and Enteral Nutrition, vol. 40, no. 2, pp. 159-211, 2016.
[7] A. Reintam Blaser, J. Starkopf, J. Starkopf et al., "Early enteral nutrition in critically ill patients: ESICM clinical practice guidelines," Intensive Care Medicine, vol. 43, no. 3, pp. 380-398, 2017.

[8] N. E. Cahill, R. Dhaliwal, A. G. Day, X. Jiang, and D. K. Heyland, "Nutrition therapy in the critical care setting: what is "best achievable" practice? An international multicenter observational study," Critical Care Medicine, vol. 38, no. 2, pp. 395-401, 2010.

[9] P. J. M. Weijs, S. N. Stapel, S. D. W. de Groot et al., "Optimal protein and energy nutrition decreases mortality in mechanically ventilated, critically ill patients," Journal of Parenteral and Enteral Nutrition, vol. 36, no. 1, pp. 60-68, 2012.

[10] S. L. Lim, K. C. B. Ong, Y. H. Chan, W. C. Loke, M. Ferguson, and L. Daniels, "Malnutrition and its impact on cost of hospitalization, length of stay, readmission and 3-year mortality," Clinical Nutrition, vol. 31, no. 3, pp. 345-350, 2012.

[11] L. L. Tian, L. Li, J. Zhao et al., "A study on the timing of enteral nutrition in postoperative patients with malignant obstructive jaundice," Parenteral \& Enteral Nutrition, no. 4, pp. 225-227+231, 2008.

[12] V. K. Vicic, M. Radman, and V. Kovacic, "Early initiation of enteral nutrition improves outcomes in burn disease," Asia Pacific Journal of Clinical Nutrition, vol. 22, no. 4, pp. 543547, 2013.

[13] Y. C. Tseng, B. C. Chen, C. T. Chen et al., "Early initiation of post-pyloric feeding in patients with major burns: experience in taiwan formosa water park dust explosion disaster," The Tohoku Journal of Experimental Medicine, vol. 247, no. 2.

[14] M. L. Stewart, M. Biddle, and T. Thomas, "Evaluation of current feeding practices in the critically ill: a retrospective chart review," Intensive and Critical Care Nursing, vol. 38, pp. 24-30, 2017.

[15] X. F. Liu, H. Q. Zhu, Q. L. Xu, and Y. M. Xie, "Comparison of two nasal-jejunal tube placement methods in critically ill patients with different acute gastrointestinal injury grades," Academic Journal of the Second Military Medical University, vol. 36, no. 09, pp. 961-965, 2015.

[16] A. R. Blaser, J. Starkopf, Ü. Kirsimägi, and A. M. Deane, "Definition, prevalence, and outcome of feeding intolerance in intensive care: a systematic review and meta-analysis," Acta Anaesthesiologica Scandinavica, vol. 58, no. 8, pp. 914-922, 2014.

[17] H. Kim, N. A. Stotts, E. S. Froelicher, M. M. Engler, and C. Porter, "Why patients in critical care do not receive adequate enteral nutrition? A review of the literature," Journal of Critical Care, vol. 27, no. 6, pp. 702-713, 2012.

[18] Z. J. Shi, H. Liu, Y. Y. Zhang, and W. Cheng, "Timing of enteral nutrition on prognosis of patients with severe acute pancreatitis: a meta-analysis," Chinese Journal of Critical Care Medicine (Electronic Edition), vol. 8, no. 2, pp. 77-83, 2015.

[19] R. Altomare, G. Damiano, A. Abruzzo et al., "Enteral nutrition support to treat malnutrition in inflammatory bowel disease," Nutrients, vol. 7, no. 4, pp. 2125-2133, 2015.

[20] X. M. Gong, X. H. Ye, and Y. Y. Xue, "Construction of early enteral nutrition tolerance assessment and management for critically ill patients," Chinese Journal of Nursing, vol. 54, no. 4, pp. 490-494, 2019.

[21] A. Reintam, P. Parm, R. Kitus, J Starkopf, and H Kern, "Gastrointestinal Failure score in critically ill patients: a prospective observational study," Critical Care (London, England), vol. 12, no. 4, pp. R90-R436, 2008. 
[22] K. Shimizu, H. Ogura, T. Asahara et al., "Gastrointestinal dysmotility is associated with altered gut flora and septic mortality in patients with severe systemic inflammatory response syndrome: a preliminary study," Neuro-Gastroenterology and Motility, vol. 23, no. 4, pp. 330-e157, 2011.

[23] N. Nguyen, K. Ching, R. Fraser, M. Chapman, and R. Holloway, "The relationship between blood glucose control and intolerance to enteral feeding during critical illness," Intensive Care Medicine, vol. 33, no. 12, pp. 2085-2092, 2007.

[24] S. Alkhawaja, C. Martin, and R. J. Butler, "Post-pyloric versus gastric tube feeding for preventing pneumonia and improving nutritional outcomes in critically ill adults," Cochrane Database of Systematic Reviews (Online), vol. 8, no. 8, p. CD008875, 2015.

[25] B. Shankar, D. K. Daphnee, N. Ramakrishnan, and R. Venkataraman, "Feasibility, safety, and outcome of very early enteral nutrition in critically ill patients: results of an observational study," Journal of Critical Care, vol. 30, no. 3, pp. 473-475, 2015.

[26] P. Fuentes Padilla, G. Martínez, R. W. Vernooij, G. Urrútia, M. Roqué I Figuls, and X. Bonfill Cosp, "Early enteral nutrition (within 48 hours) versus delayed enteral nutrition (after 48 hours) with or without supplemental parenteral nutrition in critically ill adults," Cochrane Database of Systematic Reviews, vol. 2019, no. 10, p. CD012340, 2019.

[27] F. Tian, P. T. Heighes, M. J. Allingstrup, and G. S. Doig, "Early enteral nutrition provided within 24 hours of ICU admission," Critical Care Medicine, vol. 46, no. 7, pp. 1049-1056, 2018. 